\title{
Article \\ Optimization of the Guide Design of MIRACLES, the Neutron Time-of-Flight Backscattering Spectrometer at the European Spallation Source
}

\author{
Félix J. Villacorta ${ }^{1, *(D)}$, Damián Martín Rodríguez ${ }^{2} \mathbb{D}$, Mads Bertelsen ${ }^{2}$ and Heloisa N. Bordallo ${ }^{2,3}(\mathbb{D}$ \\ 1 ESS-Bilbao, Parque Científico y Tecnológico Bizkaia 201, 48170 Zamudio, Spain \\ 2 European Spallation Source ESS ERIC, P.O. Box 176, SE-22100 Lund, Sweden; \\ Damian.Rodriguez@ess.eu (D.M.R.); mads.bertelsen@ess.eu (M.B.); bordallo@nbi.ku.dk (H.N.B.) \\ 3 Niels Bohr Institute, University of Copenhagen, Universitetsparken 5, 2100 Copenhagen, Denmark \\ * Correspondence: fjimenez@essbilbao.org
}

\section{check for}

updates

Citation: Villacorta, F.J.; Rodríguez, D.M.; Bertelsen, M.; Bordallo, H.N. Optimization of the Guide Design of MIRACLES, the Neutron

Time-of-Flight Backscattering Spectrometer at the European Spallation Source. Quantum Beam Sci. 2022, 6, 3. https://doi.org/10.3390/ qubs6010003

Academic Editors: Anna Sokolova and Akihiro Iwase

Received: 2 October 2021

Accepted: 28 December 2021

Published: 31 December 2021

Publisher's Note: MDPI stays neutral with regard to jurisdictional claims in published maps and institutional affiliations.

Copyright: (c) 2021 by the authors Licensee MDPI, Basel, Switzerland. This article is an open access article distributed under the terms and conditions of the Creative Commons Attribution (CC BY) license (https:// creativecommons.org/licenses/by/ $4.0 /)$.

\begin{abstract}
To boost the science case of MIRACLES, the time-of-flight backscattering spectrometer at the European Spallation Source (ESS), an optimized neutron guide system, is proposed. This systematic study resulted in an enhancement in the transport of cold neutrons, compared with the previous conceptual design, with wavelengths ranging from $\lambda=2 \AA$ to $20 \AA$ along the $162.5-\mathrm{m}$ distance from source to sample. This maintained the undisturbed main focus of the instrument, viz, to carry out quasielastic and inelastic neutron scattering (QENS and INS) experiments on a large dynamic range and for both energy-gain and energy-loss sides. To improve the collection of cold neutrons from the source and direct them to the sample position, the vertical geometry was adjusted to an adapted version of a ballistic elliptical profile. Its horizontal geometry was conceived to: (i) keep the high-resolution performance of the instrument, and (ii) minimize the background originating from fast and thermal neutrons. To comply with the first requirement, a narrow guide section at the pulse shaping chopper position has been implemented. To fulfil the second, a curved guide segment has been chosen to suppress neutrons with wavelengths $\lambda<2 \AA$. Subsequent tailoring of the phase space provided an efficient transport of cold neutrons along the beamline to reach a $3 \times 3 \mathrm{~cm}^{2}$ sample. Finally, additional calculations were performed to present a potential upgrade, with the exchange of the final segment, to focus on samples of approximately $1 \times 1 \mathrm{~cm}^{2}$; the proposal anticipates a flux increase of $70 \%$ in this $1 \mathrm{~cm}^{2}$ sample area.
\end{abstract}

Keywords: neutron optics; neutron instrumentation; European Spallation Source

\section{Introduction}

MIRACLES is one of the 15 scientific instruments approved for construction in the first phase of the European Spallation Source [1], anticipated to be operational before the end of this decade. MIRACLES is a neutron time-of-flight backscattering spectrometer [2,3] that will deliver a tunable energy resolution at the elastic peak (from 2 to $45 \mu \mathrm{eV}$ ), with a dynamic range, when centered around the elastic energy of the reflections of its Si (111) analyzer $(\lambda=6.27 \AA, E=2.08 \mathrm{meV})$ of nearly $\Delta E \approx 1.1 \mathrm{meV}$. The following characteristics are essential to develop the scientific performance of the instrument:

(i) large dynamical range and variable resolution will allow for the study of complex phenomena where the understanding of the dynamics at different time scales is crucial for explaining materials properties [4-6].

(ii) high energy resolution modes will allow for the investigation of slow dynamics in the order of the nanosecond and subnanosecond, which are displayed, for instance, in proteins, water in biosystems, polymers or low-lying excitations observed in molecular magnets, as well as tunneling phenomena. 
(iii) a high flux will allow handling these main experimental requirements, beyond the intuitive reduction of measurement times during an experiment.

By combining these unique characteristics, MIRACLES will offer unprecedented capabilities to groundbreaking science that requires time-resolved in-situ and in-operando experiments, the observation of dynamics in very diluted systems (a common feature in biosystems) and the possibility of discovering new phenomena hidden at the current spectrometer fluxes, such as degradation or features that display a very weak signal [7].

Since MIRACLES original concept was approved [8,9], significant upgrades to its design have been carried out. In particular, the updated chopper cascade efficiently provides a versatile performance with a very flexible selection of configuration modes [10]. Additionally, an ambitious upgrade design of the chopper layout that squeezes the time structure of the beamline may provide a pulse multiplication option for some experimental modes, e.g., for fixed-energy window scans, delivering an unprecedented flux at the sample position [11,12]. Moreover, the secondary spectrometer has reached a mature design with the improvement of the energy resolution through the optimization of the sample-analyzerdetector configuration, as well as the minimization of the background with the design of the radial collimator. Finally, to utilize the capacity of this spectrometer, the missing piece of the puzzle was to develop a detailed design of the neutron beam transport system. This is the exact scope of this work.

Similar to the long-beamline spectrometers at the ESS, which are specifically designed to take full advantage of the full pulse period ( $T=71.43 \mathrm{~ms})$, MIRACLES' length is determined to cover the complete time frame at the sample position (at $\sim 162.5 \mathrm{~m}$ ) and operate in the time-of-flight mode. This gives MIRACLES a maximum bandwidth of $\Delta \lambda=1.7 \AA$. In addition, long beamlines intuitively yield background minimization and an improvement of the energy resolution.

However, at first glance, the design of the ESS source poses two main challenges for a backscattering spectrometer. First, the long-pulse $(\Delta \tau=2.86 \mathrm{~ms})$ concept of the ESS might be considered as a less ideal feature for high-resolution modes [13-15]. Nevertheless, in the case of such a long instrument, we have demonstrated that this can be fully explored to create a versatile neutron instrument. In other words, through clever pulse tailoring, a flexible and broad range of observation times and dynamics can be selected in one experimental round $[8,10]$. Second, the reduced dimensions of the moderator, plus its long distance from the neutron beam extraction (almost $2 \mathrm{~m}$ ), inherently reduces MIRACLES phase space, and, consequently, its potential brilliance transfer. This paper reports on how this challenge was cracked.

\section{Boundary Conditions and Computational Methods}

MIRACLES is allocated in the middle of the West sector, at beamport W5, with an angle of $126^{\circ}$ with respect to the proton beam. The neutron guide starts at $L=1.9 \mathrm{~m}$ concerning the instrument coordinate system located at the west center of the ESS Butterfly cold moderator, with the optics placed within an insert that crosses the monolith wall. MIRACLES' primary spectrometer has been designed to transport cold neutrons within a wavelength ranging from 2 to $20 \AA$, from the $3 \mathrm{~cm}$ tall ESS butterfly moderator [16] to a sample size of $3 \times 3 \mathrm{~cm}^{2}$ located at $162.5 \mathrm{~m}$ from the source. This wavelength range will be employed to carry out QENS and INS measurements in both energy-gain and energy-loss sides of the inelastic spectrum with respect to the elastic energy of $E=2.08 \mathrm{meV}$ $(\lambda=6.27 \AA)$, determined by $\mathrm{Si}(111)$ backscattering analyzer reflections. To achieve the instrument requirements, MIRACLES' guide system must maximize the extraction and transport of neutrons along the beamline within the required phase space, suppressing all unwanted neutrons (i.e., background neutrons located outside the required phase space). Furthermore, it is important to keep in mind that to minimize reflection losses, the defined phase space must have low divergence along the beam trajectory. It is also important that the divergence remains wavelength independent, thus a simple guide with a constant cross-section cannot be considered. 
As a starting point, the primary spectrometer general layout consists of the parts depicted in Figure 1 and itemized below:

- Neutron beam extraction (NBEX) system, used to collect and direct the cold neutrons efficiently from the ESS butterfly moderator.

- Pulse shaping choppers area (around the PWD/PS choppers, pulse width definition and pulse selection choppers), defining MIRACLES' high-resolution mode and the primary energy resolution contribution of the TOF incident beam (at $L=7.7 \mathrm{~m}$ from the cold moderator center). The guide width at this position, as well as the horizontal profile of the beam extraction system, are limited by the location of the pulse shaping chopper.

- Curved guide, optimized to the lose direct line of sight of the source well before the sample. This section shall suppress the prompt fast neutrons and gamma photons stemming from the spallation process while keeping the cold neutrons with $E \lesssim 20 \mathrm{meV}(\lambda \geq 2 \AA)$.

- Straight section, used to transport the cold neutrons through the long guide hall to the sample with minimum loss.

- Focusing guide, designed to merge neutrons to the sample position.

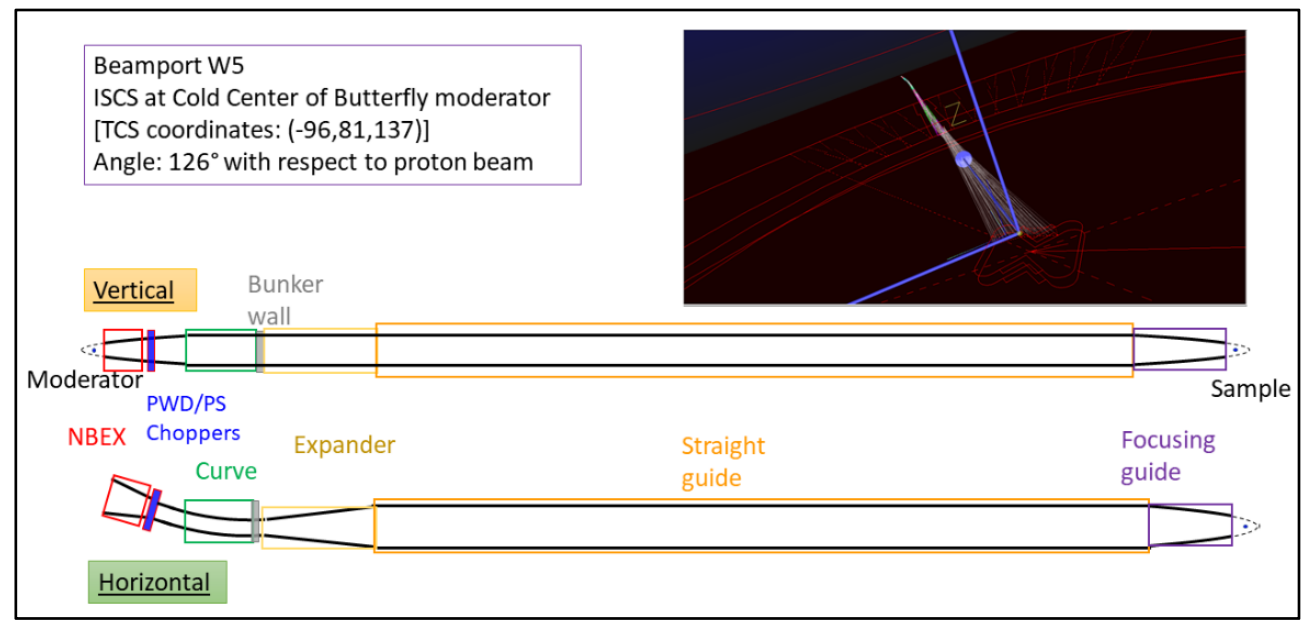

Figure 1. Vertical and horizontal layouts for the preliminary concept of the MIRACLES primary spectrometer. Inset: McStas illustration of the instrument source coordinate system, which is located at the west corner of the cold source of the ESS butterfly moderator.

Following simple analytical calculations, subsequent systematic Monte Carlo raytracing simulations were carried out using McStas (version 2.4) [17], where the source was modeled using the ESS_butterfly moderator component [18]. Calculations check the quality of the overall spectrum, i.e., carried out with the choppers at the park-open position, and implementing the gaps corresponding to these choppers for more realistic results. For all explored guide configurations, eight ESS pulses were simulated, using both Guide_gravity and Elliptic_guide_gravity components. The flux at different positions along the guide was monitored by placing different monitors components (Monitor_nD, PSD_monitor, L_monitor and TOF_monitor) at selected positions. Results have been compared to the intensity on the $3 \times 3 \mathrm{~cm}^{2}$ sample. 


\section{Results and Discussion}

\subsection{Neutron Beam Extraction (NBEX) and Pulse Shaping Section}

The instrument source coordinate system (ISCS, i.e., the origin) for MIRACLES is located at the west corner center of the cold moderator (as illustrated in the inset of Figures 1 and 2a). The viewable width of the cold moderator surface in the MIRACLES horizontal layout extraction system is approximately $70 \mathrm{~mm}[18,19]$; this defines the maximum width of the horizontal aperture. Furthermore, MIRACLES has an additional restriction related to the consecution of high-resolution modes. To achieve short pulses (down to $60 \mu \mathrm{s}$ ), the pulse-shaping chopper slits need to be narrow, a feature that limits the guide section at the pulse shaping chopper position (width, $w=54.6 \mathrm{~mm}$ ) [10]. Accordingly, the initial phase space volume (defined as the efficiency of the beam extraction system) will depend on the distance between the source and the guide entrance, their dimensions and the reflectivity profile of the guide.

Starting from this latter feature, the first constraint for the NBEX is the supermirror value, which was set to $m=4$ (with a reflectivity value close to the critical angle of $R=0.79$ ) for the horizontal and vertical layouts. Here we note that, from the point of view of optical performance, no significant improvement in the collection of cold neutrons is expected by using higher supermirror values in the neutron beam extraction segment.

Several horizontal apertures for the NBEX, from 60 to $70 \mathrm{~mm}$, were tested. Results revealed that a horizontal aperture larger than $66 \mathrm{~mm}$ (Figure 2b) does not increase the intensity at the exit of the pulse shaping choppers (which defines the exit of the neutron beam extraction area); hence, a tapered convergent horizontal profile was selected, starting with a width of $66 \mathrm{~mm}$ at the guide entrance $(L=1.9 \mathrm{~m})$ and finishing with a width of $54.6 \mathrm{~mm}$ at the pulse shaping chopper position $(L=7.7 \mathrm{~m})$.

The vertical layout can take advantage of a ballistic profile to minimize reflection losses [20]. However, the vertical dimensions of the beam extraction segment are restricted by the housing of the monolith insert (named neutron beam port insert, NBPI), imposing a maximum value for this vertical section at the exit of the insert of $71.5 \mathrm{~mm}$. Considering this constraint, two main options for the elliptical profile were tested: one with foci located at $f_{1}=0 \mathrm{~m}$ and $f_{2}=16 \mathrm{~m}$, with the center of the ellipse coinciding approximately with the pulse-shaping chopper system, and the other with the second focus located at the sample position, i.e., $f_{1}=0 \mathrm{~m}$ and $f_{2}=162.5 \mathrm{~m}$. The latter option shows a narrower angular divergence distribution of the transported neutrons (see Figure 3a), which will benefit their downstream transport throughout the guide. Afterwards, this option was further optimized during the simulations, as explained below.

\section{Neutron beam extraction: Horizontal layout}

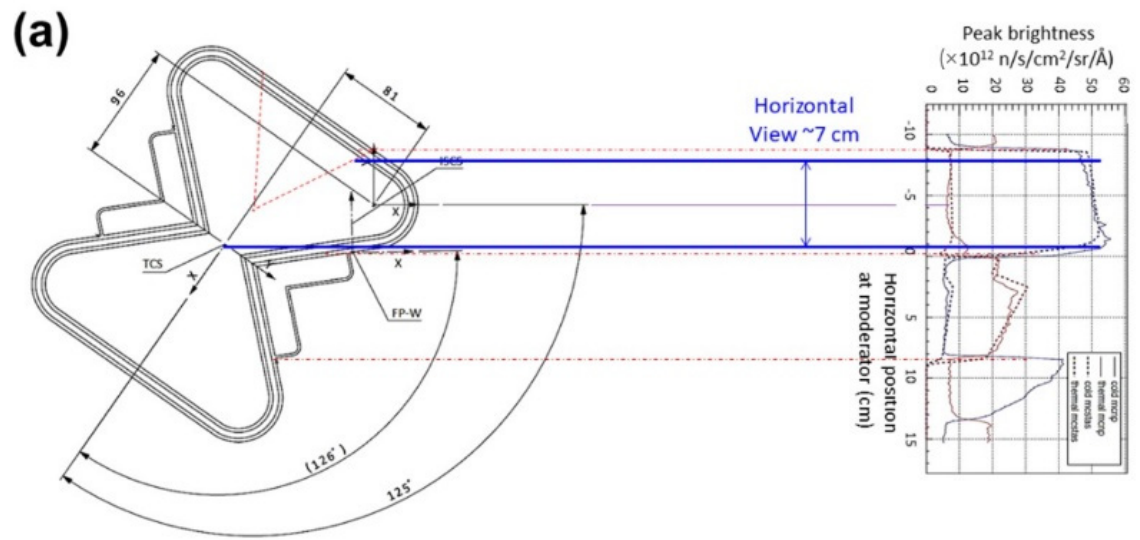

Figure 2. Cont. 


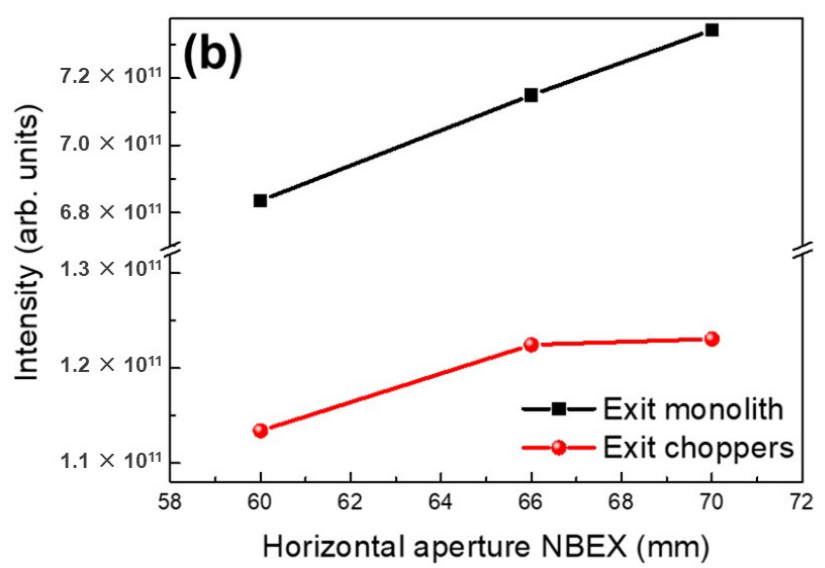

Figure 2. (a) Horizontal view of the moderator adapted from Ref. [18]; (b) Integrated intensity monitored at the exit of the NBEX and the end of the curved guide for several widths: $60 \mathrm{~mm}, 66 \mathrm{~mm}$ and $70 \mathrm{~mm}$.
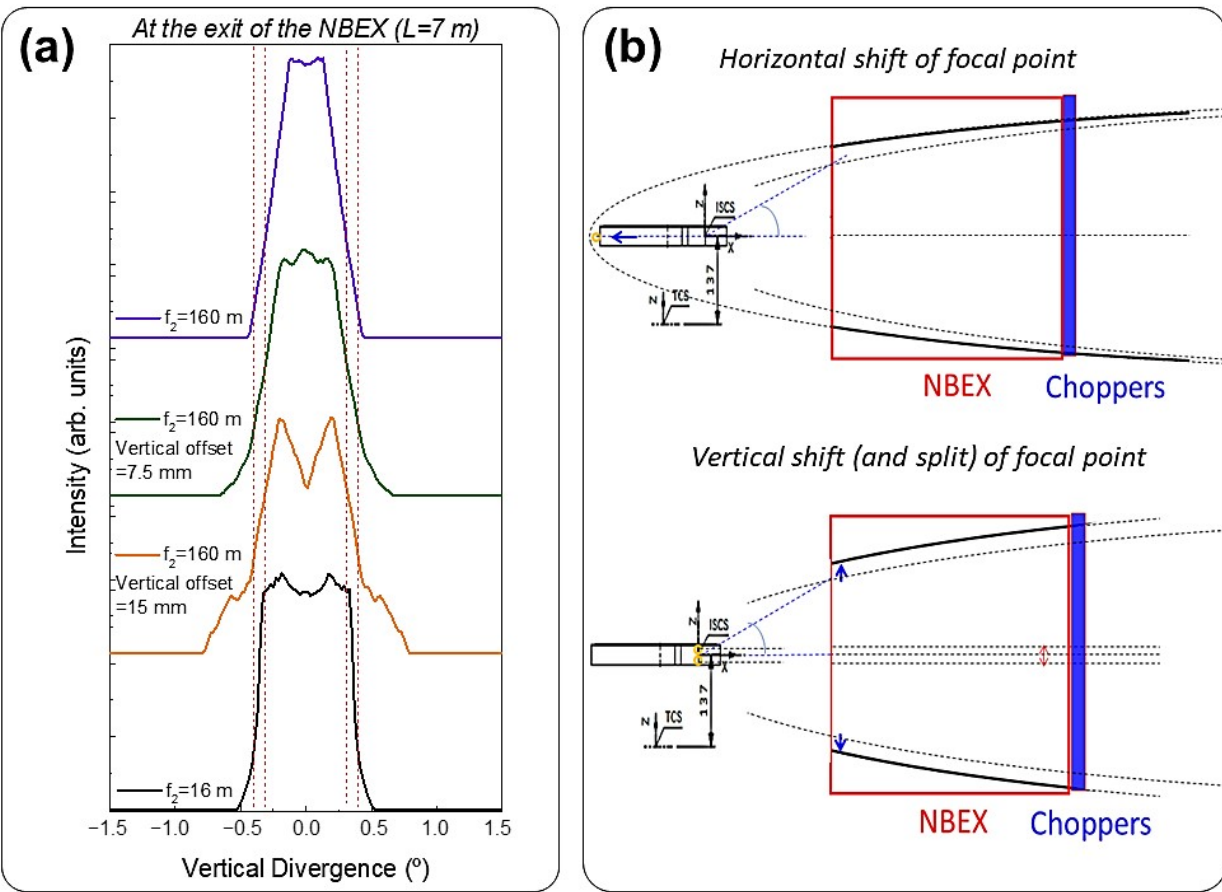

Vertical shift (and split) of focal point

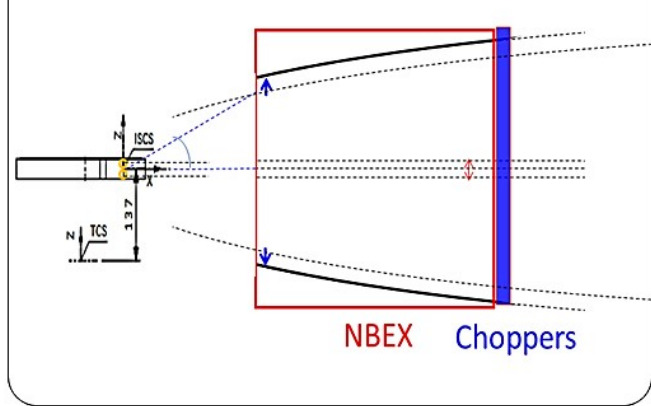

Figure 3. (a) Vertical divergence distributions considering the vertical design options described in the text; (b) Schematic layout of the options considered for the vertical profile of the NBEX, which included a horizontal shift of the focal point (yellow dot) upstream to the cold source (top image), and a vertical shift and split of the focal point (yellow dots, bottom image).

Before we proceed with the optimization of MIRACLES' NBEX, it should be highlighted that the NBEX for all ESS instruments starts quite far from the moderator, at $L \approx 1.9$ $\mathrm{m}$. This circumstance reduces the available phase space volume to be transported along the beamline guide system. For a spectrometer such as MIRACLES, a large phase space volume can be accepted since divergence is not a critical issue at the sample position, and guide aperture dimensions can be expanded to somewhat improve the neutron collection and final flux at the sample. Thus, to extract and transport the desired neutrons, $\lambda \geq 2 \AA$, and assuming the supermirror value of $m=4$, the maximum achievable critical angle is $0.8^{\circ}$, $\theta_{C}=0.1 \times m \times \lambda$. This implies that for a guide located at $L=1.9 \mathrm{~m}$ from the cold source, the required vertical aperture to collect those neutrons is $\sim 53 \mathrm{~mm}$, larger than the entrance of the elliptic profile when $f_{2}=162.5 \mathrm{~m}$, that is $\sim 42 \mathrm{~mm}$. Therefore, there is room for an increase of the vertical guide aperture at the entrance of the beam extraction to improve the 
collection of neutrons produced at the moderator towards the sample. Accordingly, two methodologies that relax the pure point-like source approach to defocus the ellipse with respect to the source have been considered (see Figure 3b), consisting of:

(a) shifting the focus horizontally behind the cold source, or

(b) shifting and splitting the focus vertically into two focal points symmetric to the center plane of the moderator up and down.

The first option shows good results by locating the $f_{1}$ point $1 \mathrm{~m}$ behind the center of the cold moderator's west corner. This layout provides an increase in the collection of useful neutrons as a result of a larger guide entrance (up to $15 \%$ with a vertical aperture of $\sim 49 \mathrm{~mm}$ ). The second option was tested considering several offset values and assuming the mechanical restrictions previously mentioned. A convenient trade-off between flux and beam divergence distribution at the NBEX exit was obtained with a vertical offset of $7.5 \mathrm{~mm}$, yielding an aperture of $\sim 49 \mathrm{~mm}$ at the guide entrance and a similar increase in the neutron collection. A higher offset displays a bimodal and larger vertical divergence distribution at the exit of the NBEX. This becomes clear when we compare, for example, the divergence distribution for vertical offsets of 7.5 and $15 \mathrm{~mm}$ above and below the guide axis (Figure 3a).

Finally, it has been observed that the rotation of the NBEX to negative angles yields a significant increase of the total surface of the cold moderator "viewed" by the extraction system and subsequently a considerable increase of the total neutron flux (see Figure 4). As construction constraints impose a limit for the rotation angle to $\varphi=-1.0^{\circ}$ (a higher deviation will exceed the allocated monolith beam port space dedicated to MIRACLES), the final angle of the MIRACLES beamline with respect to the proton beam was reduced and fixed at $125^{\circ}$.

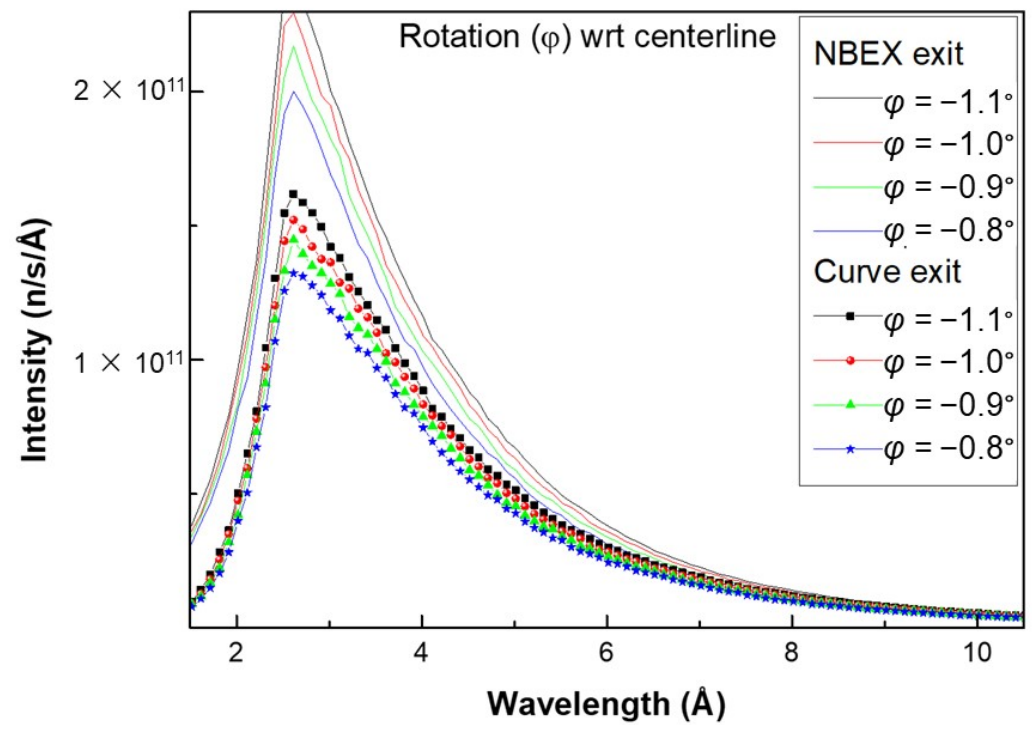

Figure 4. Spectra profile at the exit of the $\operatorname{NBEX}(7.7 \mathrm{~m}$ from the moderator) and at the exit of the curved guide ( $37 \mathrm{~m}$ from the moderator, assuming a curve with radius $R=1400 \mathrm{~m}$ ) for different rotation angles.

\subsection{Curved Guide Section}

After the pulse shaping or pulse width definition (PWD) chopper, located at $L=7.7 \mathrm{~m}$, the horizontal guide dimensions are expanded until the horizontal curved guide section, which was designed to avoid the direct line of sight of the source [21]. The selection of a horizontal curvature instead of a vertical one is based on two reasons: (i) this approach is less complex, simply because by progressively changing the height of the guide, different guide supports would need to be manufactured; (ii) horizontal curving is less sensitive to floor movements. 
The curvature acts as a filter that rejects all thermal and fast neutrons with wavelength $\lambda<2 \AA$ stemming from the source, thus minimizing the arrival of background neutrons to the sample and detectors. However, the concept of loss of direct line of sight of the source must be extended to components with a direct view of the source (choppers, fission chamber). These components are illuminated with high energy hadrons from the source that may generate showers of secondary particles, subproducts and scattered thermal neutrons; thus, avoiding direct view of these "secondary sources". Starting the calculation of the loss of the direct line of sight from this point and not from the moderator is a convenient strategy [19].

The final consideration of this guide section results from a trade-off between the optimization of outgoing flux at the exit of the curved guide and the final cost of the instrument due to beamline shielding and supermirror coating of the guides. In addition, to reduce the final cost of the beamline shielding, it is intended that all or most of the curve length should lie within the bunker, defined as the common shielding area for all instruments in their area close to the monolith, where the density of instrumentation is significant. However, this should not be at the expense of a significant reduction of the total outgoing flux caused by the selection of a small radius of curvature.

The optimization was carried out as follows. The m-value from the exterior lateral supermirror of the curve was fixed to $m_{\text {ext }}=2.5$; additional calculations performed using $m_{\text {ext }}=3$ gave no significant improvement in the outgoing flux. For the inner lateral guide wall and the top and bottom ones, a supermirror value of $m=2$ is more than enough to ensure an efficient neutron transport. The cut-off wavelength $\left(\lambda_{C}=2 \AA\right)$ and the supermirror value $\left(m_{\text {ext }}=2.5\right)$ are input values for the characteristic angle of a curved guide, $\gamma_{C}$, that defines its capability to lose direct line of sight from the source:

$$
\lambda_{C}=\frac{4 \pi}{m Q_{c, N i}} \gamma_{C}=\frac{574}{m} \sqrt{\frac{2 w}{R}}
$$

where $Q_{c, N i}=0.218 \AA^{-1}$ is the momentum transferred in the reflection of a neutron by a nickel layer $(m=1), m$ the supermirror value, and $R$ and $w$ define the radius and width of the guide, respectively. Thus, the loss of direct line of sight is then observed at a distance $L_{\text {curve }}$ :

$$
L_{\text {curve }}=2 R \gamma_{C}=\sqrt{8 w R}
$$

Focusing the impact of $L_{\text {curve }}$ and radius of curvature, $R$, in the performance and layout of the spectrometer, while considering construction restrictions within the ESS facility, several combinations have been tested:

(a) Entire curve within the bunker $\left(L_{\text {curve }}=16 \mathrm{~m}, R=775 \mathrm{~m}\right.$ and $\left.w=52.5 \mathrm{~mm}\right)$.

(b) Curve mostly within the bunker and slightly extended through the bunker wall and guide hall $\left(L_{\text {curve }}=26 \mathrm{~m}, R=1400 \mathrm{~m}\right.$ and $\left.w=60 \mathrm{~mm}\right)$.

(c) Half of the curve within the bunker + half in the guide hall ( $L_{\text {curve }}=36 \mathrm{~m}, R=2025 \mathrm{~m}$ and $w=80 \mathrm{~mm}$ ).

Other options with a larger radius (such as the one shown in Figure 5, with $R=3150 \mathrm{~m}$, or the one proposed in the original concept, $R=5000 \mathrm{~m}$ ) [8] were ruled out because of the excessive shielding cost required to obtain a small flux improvement (Figure 5) and construction issues (i.e., the beamline exceeds the space assigned for the instrument).

The best choice, in terms of flux at the exit of the curve and cost optimization, was found to be option (c), with $R=2025 \mathrm{~m}, w=80 \mathrm{~mm}$ and $L=36 \mathrm{~m}$. Reducing the radius of curvature to get out of the direct line of sight into the bunker results in a significant decrease of the total flux, mainly near the maximum of the cold moderator spectral range (at $\lambda \sim 3.2 \AA$, Figure 5), which is a region of particular interest for MIRACLES to perform INS measurements and QENS measurements at higher energies envisioned in future upgrades. 


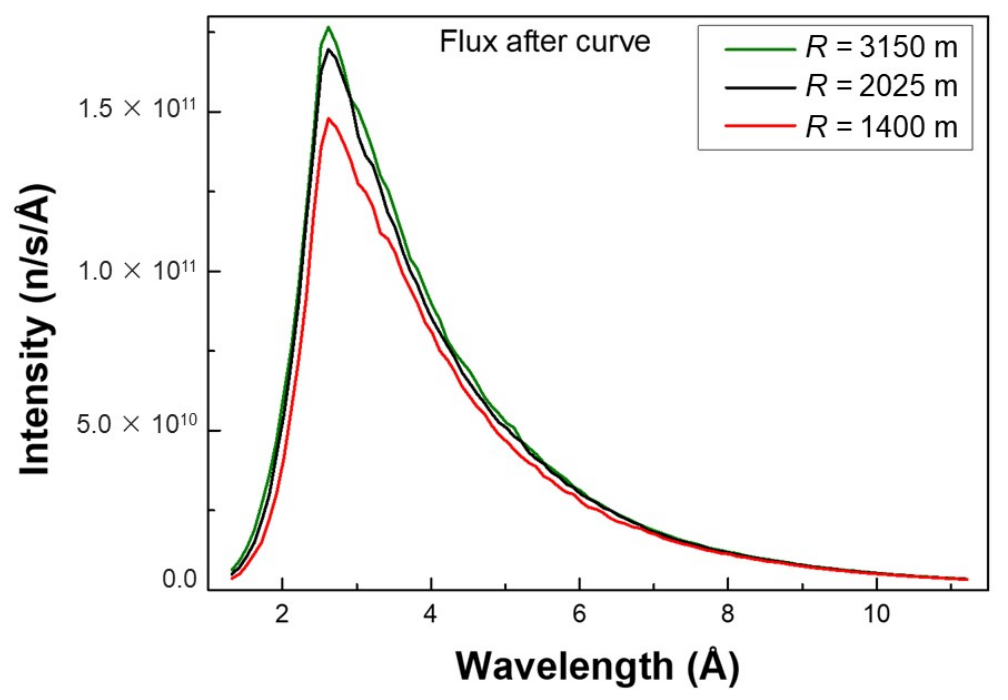

Figure 5. Spectral intensity distribution for the curved guide options described in the text.

\subsection{Long Straight Guide Section}

The compression in the horizontal guide section at the neutron beam extraction down to the pulse shaping chopper position, due to compliance with the high-resolution characteristics of MIRACLES, in addition to the curved guide, causes an unavoidably large horizontal divergence distribution at the exit of the curve (see Figure 6a). This affects the transmission of the cold neutrons between the exit of the curve and the sample (separated by $\sim 115 \mathrm{~m})$.

A divergent tapered guide segment (expander) implemented after the curve will decompress the spatial dimensions progressively in the horizontal layout (see Figure 6b), resulting in the concomitant narrowing of the horizontal divergence distribution $\Delta \phi^{H}$. This fully agrees with Liouville's theorem, which suggests an invariant phase space volume in the transport process, defined as:

$$
I \sim \Delta A \Delta \phi \Delta \lambda=\Delta x \Delta y \Delta \phi^{H} \Delta \phi^{V} \Delta \lambda
$$

where $\Delta A=\Delta x \Delta y$ is the beam dimension, $\Delta \phi^{H}$ and $\Delta \phi^{V}$ are horizontal and vertical angular divergence distributions, respectively, and $\Delta \lambda$ is the wavelength bandwidth.

To transport efficiently cold neutrons with $\lambda \geq 2 \AA$ using a maximum supermirror value for the long straight segment of $m=1.5$, we must have $\Delta \phi^{H}= \pm 0.3^{\circ}$. This can be achieved by expanding the guide width from $w=80 \mathrm{~mm}$ to $w=130 \mathrm{~mm}$ in a guide segment of $L=15 \mathrm{~m}$ (thus a tilt angle of the horizontal guide walls to provide the expansion of $0.1^{\circ}$, lower than the divergence distribution width, in both left and right guide walls). This reduces the horizontal divergence distribution width from $\Delta \phi_{F W H M}^{H}= \pm 0.5^{\circ}$ to $\Delta \phi_{F W H M}^{H}$ $= \pm 0.3^{\circ}$, also displaying a wavelength-independent profile in the horizontal and vertical directions, as shown in Figure 6. A small increase in the vertical dimensions, which has an intrinsic narrow vertical divergence distribution, from $w=100 \mathrm{~mm}$ to $w=110 \mathrm{~mm}$, results in slightly higher flux. The consequence of this tailoring of the divergence distribution is an efficient transport of neutrons, with an overall transmission of more than $90 \%$ of neutrons from the end of the curved guide to the entrance of the focusing guide along the nearly $100 \mathrm{~m}$ of this straight guide segment (see Figure 7). 

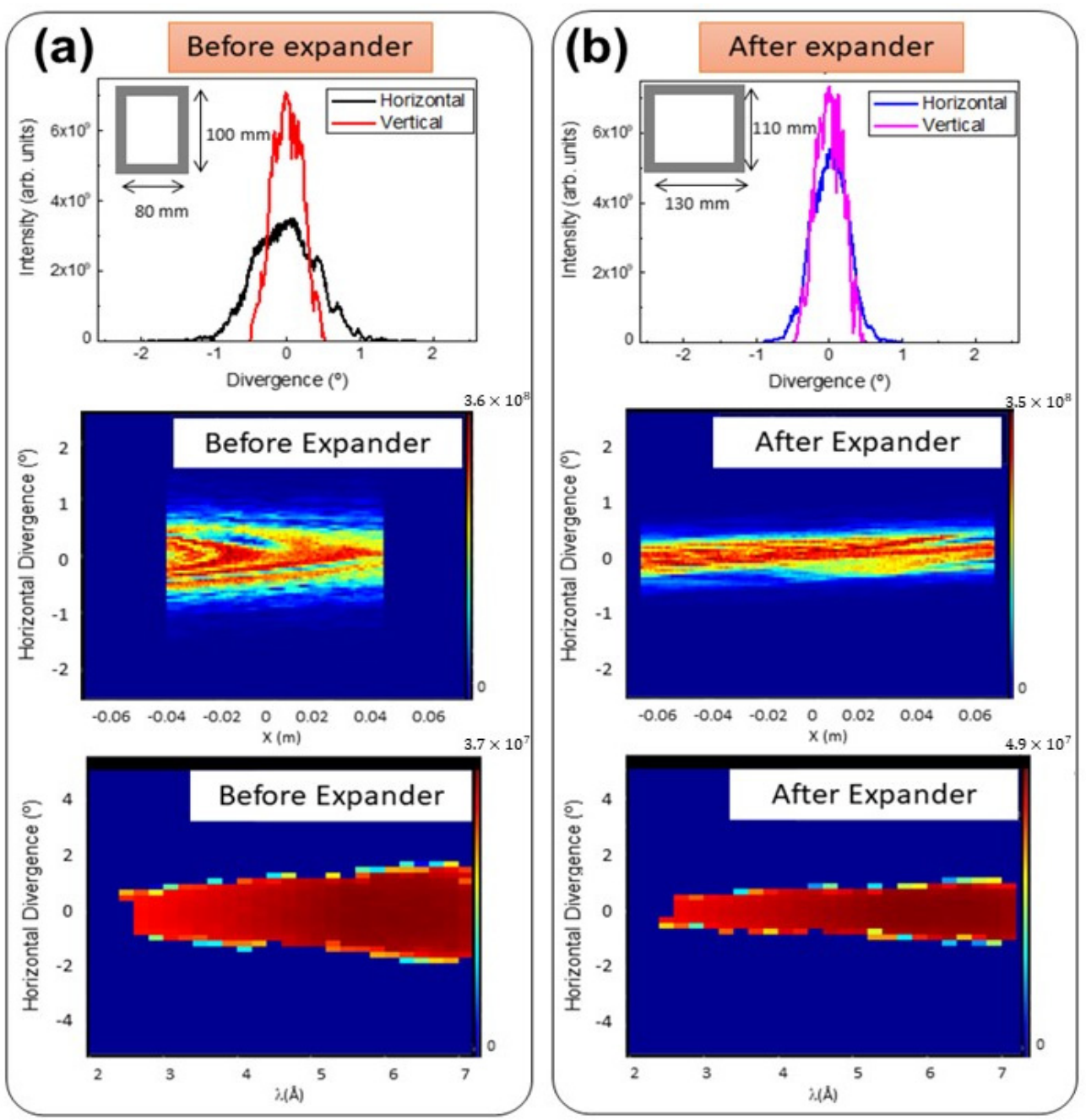

Figure 6. Overall divergence distribution (top) and acceptance diagrams with the horizontal divergence distribution as a function of position (middle) and wavelength (bottom), (a) before the expander and (b) after the expander. The grey rectangle represents the guide section at every position.

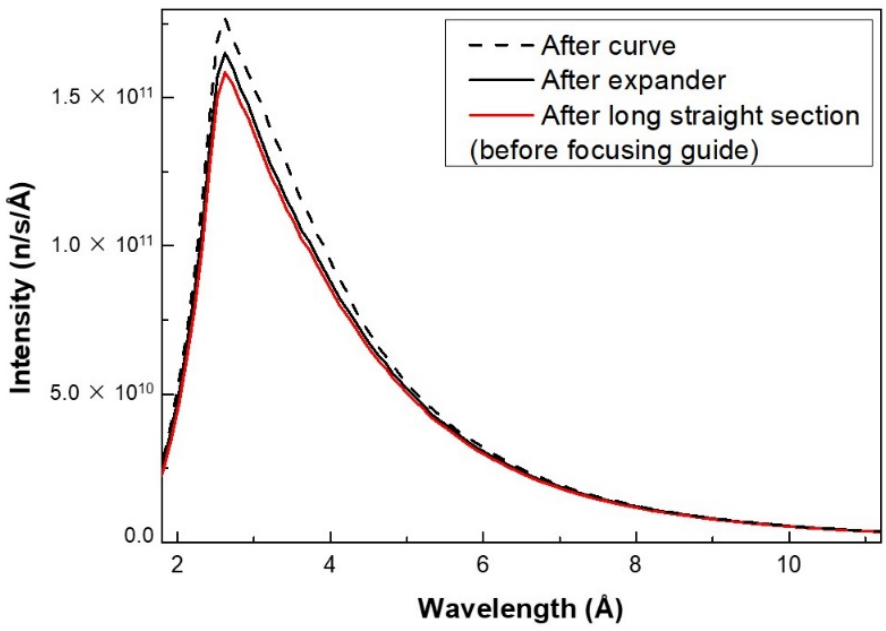

Figure 7. Spectra comparing the intensity at the entrance and exit of the long straight section.

As mentioned above, MIRACLES' optics are optimized to transmit neutrons within a wavelength range of 2 to $20 \AA$. However, with the current design, even if MIRACLES' chopper cascade removes most of the previously reported long-wavelength contaminant 
pulse at $\lambda \sim 35-42 \AA$ [10], very low-intensity tails of this unwanted feature are still present. To remove such neutrons, a frame overlap mirror (FOM) was conceptually designed, located at the end of the long straight section, with an inclination of $\alpha \sim 3.75^{\circ}$ and a coating of $m=1.5$, ensuring a cut-off wavelength at approximately $\lambda \sim 25 \AA$ [10]. A frame overlap mirror has been chosen over an additional chopper since (i) it is cheaper and (ii) it requires low or no maintenance. Monte Carlo calculations (using the mirror component in the McStas code) confirm a reduction of the long-wavelength neutron flux at $\lambda \sim 35-42 \AA$ by three orders of magnitude (Figure $8 \mathrm{a}$ ). This results in a major suppression of the contaminant pulse at $\lambda \sim 35-42 \AA$ when the chopper cascade selects the elastic wavelength of $\lambda=6.27 \AA$ at its high-resolution mode (Figure 8 b).

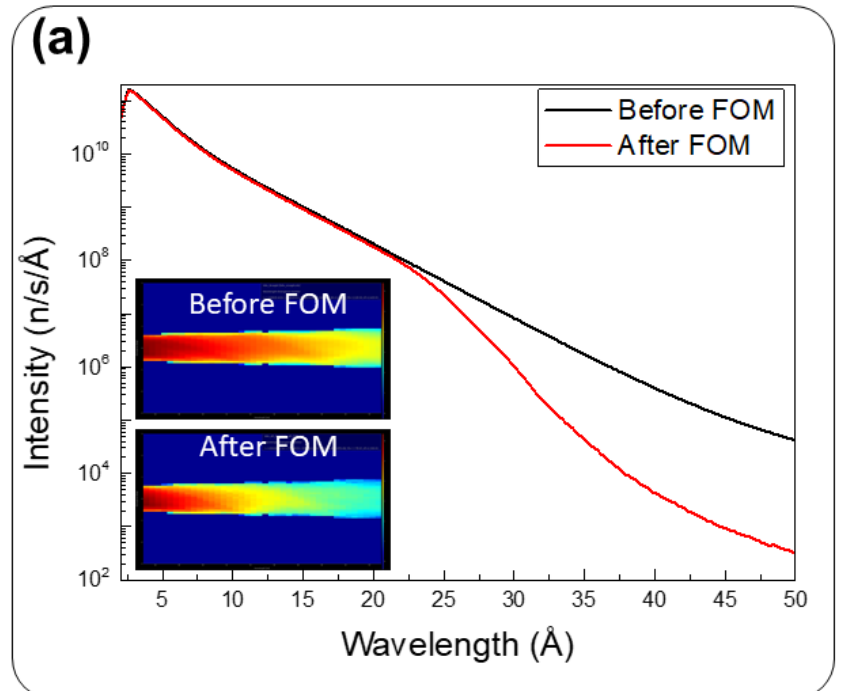

\section{(b)}

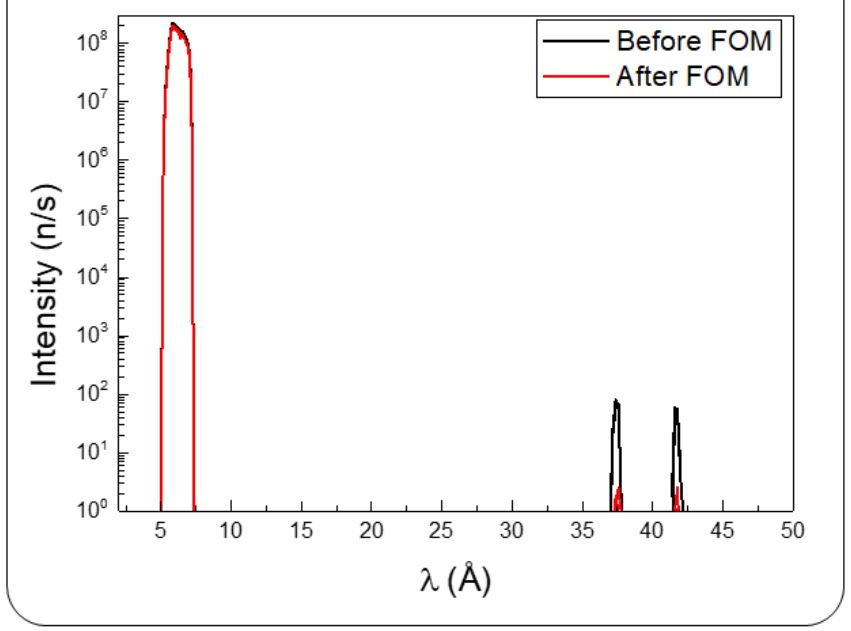

Figure 8. (a) Effect of the reduction of the long-wavelength neutrons by setting a frame overlap mirror with a cut-off wavelength of about $\lambda \sim 25 \AA$. Inset: the wavelength dependence of neutron flux and vertical divergence before and after the frame overlap mirror (FOM); the scales are $\lambda=[25$, 45] $\AA$ for the x-axes, $\Delta \phi^{V}=[-3,3]^{\circ}$ for the y-axes and $[0,6] \cdot 10^{6} \mathrm{n} / \mathrm{s}$ for the color scale. (b) Same case for choppers running at the high-resolution mode $(f=224 \mathrm{~Hz}, \delta E=2 \mu \mathrm{eV})$, tuned for $\lambda=6.27 \AA$.

\subsection{Focusing Guide Section}

MIRACLES, as most neutron spectrometers, can take advantage of a relaxed divergence and consequent increase of the final flux at the sample position. As a result of this strategy, the focusing guide must extend to as close as possible to the sample. Nevertheless, due to the dimensions of the sample environment equipment and the implementation of slits and a normalization beam monitor between the guide and sample environment, the guide must end at about $30 \mathrm{~cm}$ before the $3 \times 3 \mathrm{~cm}^{2}$ sample.

The design of the focusing part of the guide follows the design principles outlined in Ref. [22]. This work shows how the beam characteristics change depending on the shape of the focusing ellipse and the incoming beam's divergence. As the phase space of the beam before the focusing guide section is square in both dimensions and wavelengthindependent, we ensured that the beam characteristics after the focusing beam would be the desired ones independent of the wavelength chosen for the experiment.

Elliptic guides focused on, or near, the sample position is the preferred choice, and several options were considered here (illustrative images of the focusing guide profiles are shown in Figure 9):

(a) 1st option, elliptic profile, with focus $f_{2}$ at the sample, with a semiaxis value of $a=17 \mathrm{~m}$ (length of the focusing guide).

(b) 2nd option, elliptic profile with foci at the sample and the cold source ( $a=162.5 \mathrm{~m}$ ). 
(c) 3rd option, using the same elliptic profile as the 2nd option ( $a=162.5 \mathrm{~m})$, but shifting the focusing guide by displacing the geometrical focal point behind the sample position, assuming that the geometrical focal point and the effective focal point (defined as the point where the neutron flux is higher) are not the same, (i.e., thus aimed to a correction of coma aberration) [22]. This option is similar to the horizontal defocusing proposed for the NBEX.

(d) 4th option, using the same elliptic profile as the 2 nd option ( $a=162.5 \mathrm{~m}$ ), but with vertical shift and split of the focal points for top and bottom guide walls to open the guide and provide a more uniform flux at the extension of the sample $\left(3 \times 3 \mathrm{~cm}^{2}\right)$. This option is similar to the vertical defocusing proposed for the neutron beam extraction.
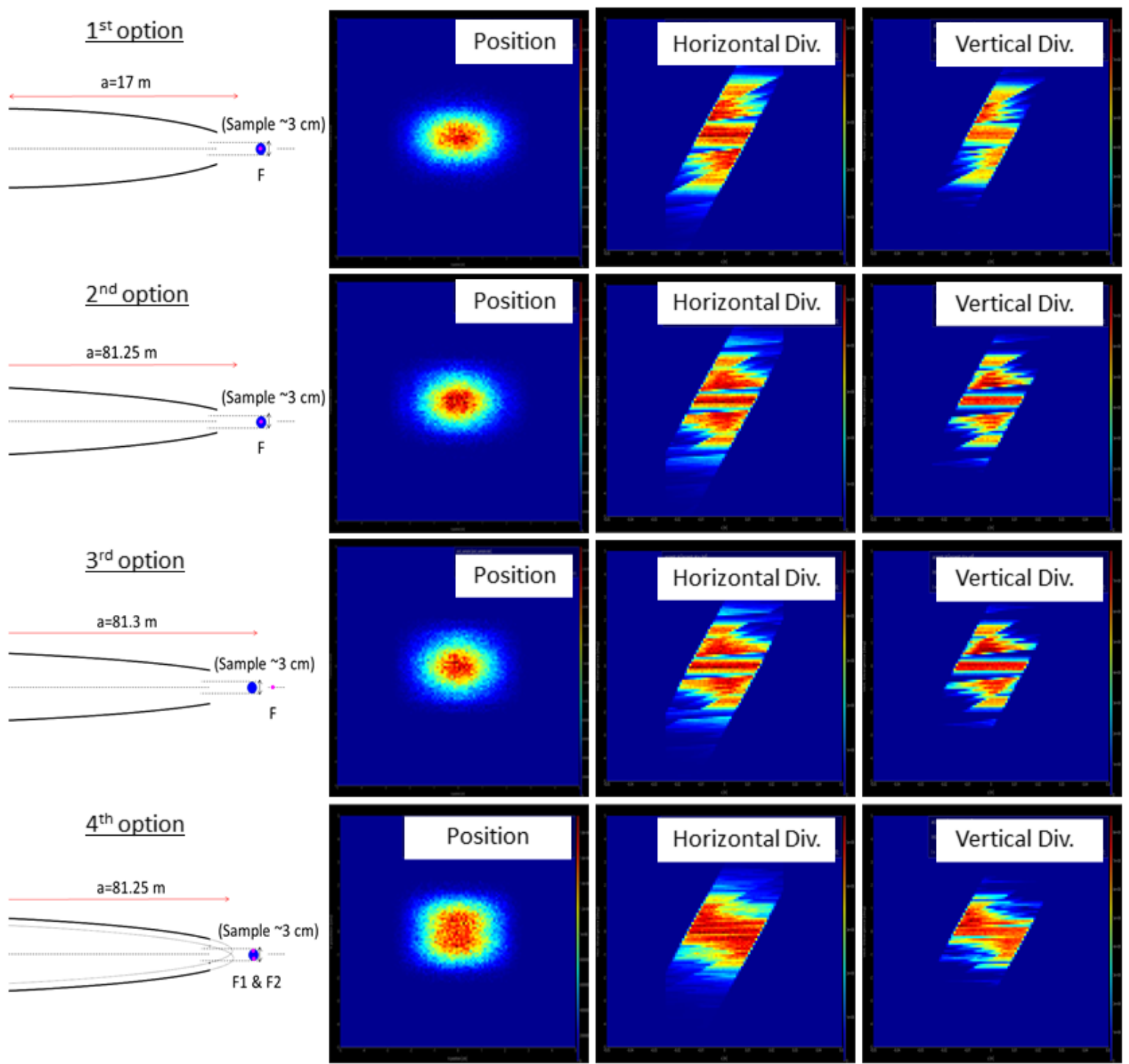

Figure 9. Beamspots and acceptance diagrams (position-divergence plots) for the four focusing guide options described in the text. For all configurations, the range of the divergence axes is $\pm 5^{\circ}$, the position axes $\pm 5 \mathrm{~cm}$, and $[0,5] \cdot 10^{8} \mathrm{n} / \mathrm{s}$ and $[0,7] \cdot 10^{8} \mathrm{n} / \mathrm{s}$ for the color scale of the horizontal and vertical diagrams, respectively.

All the preliminary calculations were carried out using a homogeneous supermirror value for the focusing guide with $m=4$ and a focusing guide within a length ranging from 9 to $17 \mathrm{~m}$, depending on the option proposed. Further optimizations give the same intensity at the sample with a gradual distribution of the m-value, from 2 to 4 , with $m=5$ 
in the last segment of the focusing guide (see Table 1). Results presented in Figure 9 reveal a very homogeneous spatial distribution of the neutron beam spot with a full width at half maximum of $\sim 3 \mathrm{~cm}$ diameter for all options. Moreover, the acceptance diagrams at the sample position reveal that approximately $99 \%$ of the neutrons are confined within a divergence range of $\Delta \phi \sim \pm 2.5^{\circ}$. In the first three options, the direct view and reflected parts in the phase space are not overlapped, which leads to gaps in the phase space. This is a usual feature in elliptical focusing guides [23]. In the fourth option, however, there are no gaps, and both contributions overlap, giving a smoother divergence distribution.

Table 1. Coating for the focusing system (both $3 \times 3 \mathrm{~cm}^{2}$ sample size, option 1 , and $1 \times 1 \mathrm{~cm}^{2}$ sample size), distance is measured from the end of the long straight section.

\begin{tabular}{cccc}
\hline Start Distance $(\mathbf{m})$ & End Distance $(\mathbf{m})$ & $\boldsymbol{m}$ Top/Bottom & $\boldsymbol{m}$ Left/Right \\
\hline 0 & 8.0 & 2 & 2 \\
8.0 & 13.0 & 2 & 2 \\
13.0 & 14.5 & 2.5 & 2.5 \\
14.5 & 15.5 & 3 & 3 \\
15.5 & 16.0 & 4 & 4 \\
16.0 & 16.8 & 5 & 5 \\
\hline
\end{tabular}

Even if the guide system has been optimized for the large sample size of $3 \times 3 \mathrm{~cm}^{2}$, the capability to focus on a smaller sample size of $1 \times 1 \mathrm{~cm}^{2}$ is already foreseen as a future upgrade. Hence, the feasibility of having two different focusing noses on the MIRACLES guide system for two different sample sizes was analyzed. The exchangeable guide cannot be implemented to the total length of this focusing section. An alternative consists of the design of an aggressive option for the last meters where the guide segment is embedded within the scattering vessel (see Figure 10a).

Therefore, the focusing section was split into three components, first the main elliptic section connects to the long straight guide and continues to the exchangeable section. The exchangeable section was then simulated with two different components with independent geometrical parameters. Both exchangeable elements are required to have the same dimensions at the crossover to the main ellipse so that the guide is continuous regardless of which focusing element is chosen. In the simulation, each neutron selects a random focusing element to activate, i.e., each neutron randomly chooses between the two noses with a 50\% probability for each; in this way, each is weighted the same in the figure of merit and, both are simulated in a single McStas run. In the calculations, the focusing layout labeled as Option 1 was chosen for the $3 \times 3 \mathrm{~cm}^{2}$ focusing nose. The same coating distribution from this version is used on the $1 \times 1 \mathrm{~cm}^{2}$ elliptic guide elements and is described in Table 1 .

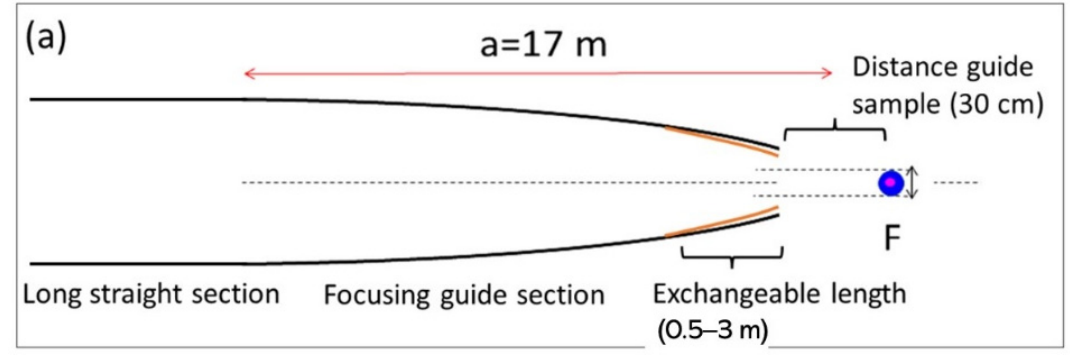

Figure 10. Cont. 

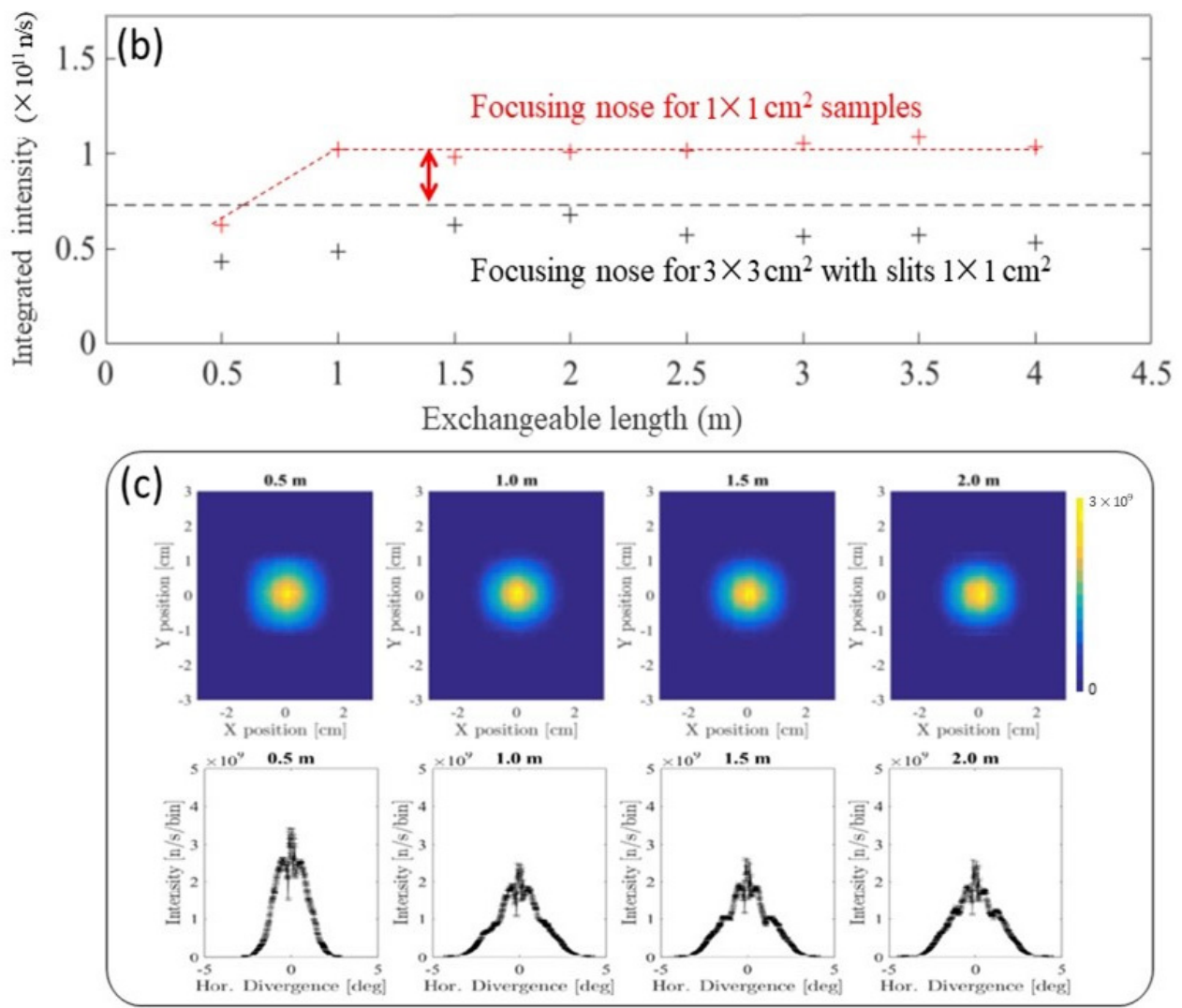

Figure 10. (a) Schematic image illustrating the exchangeable focusing guide concept; (b) Integrated intensity at sample position using a focusing guide for $3 \times 3 \mathrm{~cm}^{2}$ beam spot with slits aperture of $1 \times 1 \mathrm{~cm}^{2}$ (black dots), compared with the intensity using an exchangeable focusing nose with variable length for $1 \times 1 \mathrm{~cm}^{2}$ beam spot (red dots); (c) Beamspots (spatial distributions) and horizontal divergence distributions at the sample position for different exchangeable focusing guide lengths (from 0.5 to $2 \mathrm{~m}$ ). The vertical divergence distributions are not shown since the profile is similar to the horizontal counterparts.

Figure 10b show the total flux as a function of the length of the exchangeable part for the two configurations: (i) focusing guide for $3 \times 3 \mathrm{~cm}^{2}$ samples, with slits opened by $1 \times 1 \mathrm{~cm}^{2}$, and (ii) focusing nose for $1 \times 1 \mathrm{~cm}^{2}$. Results confirm a significant increase of flux when compared to the option of exchange only the last $1 \mathrm{~m}$ of the $17 \mathrm{~m}$ long elliptical focusing guide. Moreover, a longer exchangeable element does not provide further benefit. This is a very convenient result from the point of view of mechanical design since the exchangeable focusing guide cartridge can fit inside the scattering vessel. The beam spots (Figure 10c) are smooth and homogeneous in all cases. As expected, the horizontal divergence distributions are slightly broader than for the $3 \times 3 \mathrm{~cm}^{2}$ case. The exchangeable versions with perfect elliptic mirrors are smoother but show a spike at 0 divergences in the vertical and horizontal profiles.

Figure 11a show the spectra at the sample position for the two configurations, (i) the focusing guide for $3 \times 3 \mathrm{~cm}^{2}$ samples with slits opened by $1 \times 1 \mathrm{~cm}^{2}$, and (ii) focusing nose for $1 \times 1 \mathrm{~cm}^{2}$. The ratio between both spectra (Figure 11b) reveals a superior (and wavelength-dependent) performance of the second option, displaying a gain of approximately $70 \%$ at the $6.27 \AA$ wavelength for the focusing nose for $1 \times 1 \mathrm{~cm}^{2}$ with respect to the first one. 


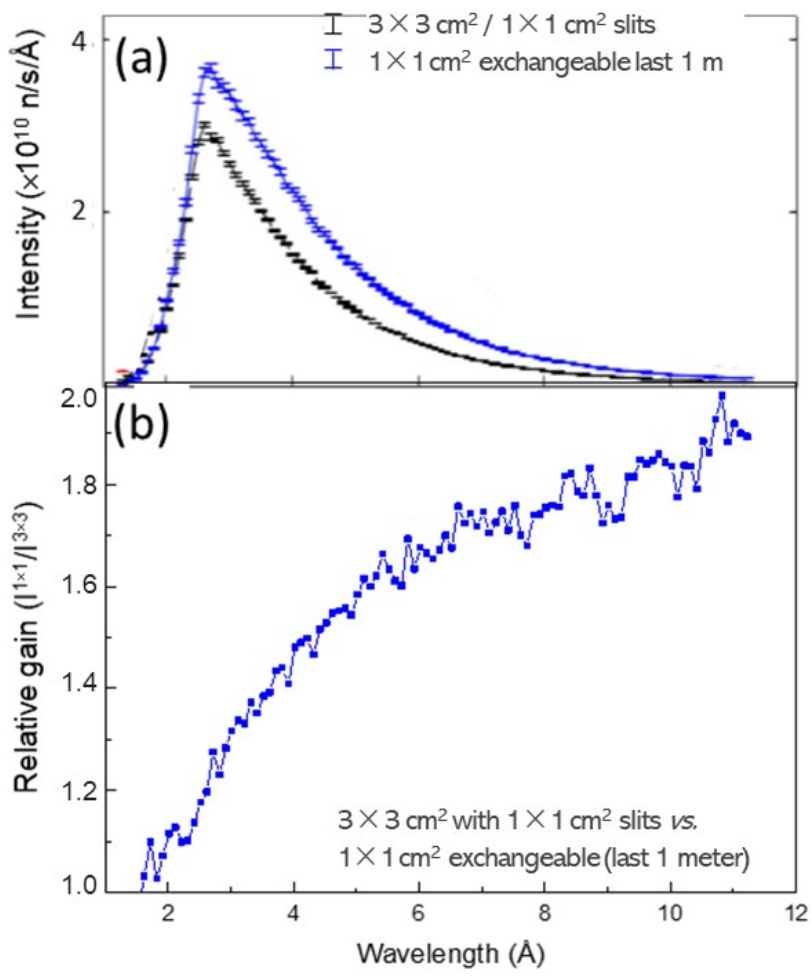

Figure 11. (a) Spectra corresponding to the 2 configurations (current focusing guide for $3 \times 3 \mathrm{~cm}^{2}$ samples, with slits opened by $1 \times 1 \mathrm{~cm}^{2}$, and focusing nose for $1 \times 1 \mathrm{~cm}^{2}$, with an exchangeable section in the last $1 \mathrm{~m}$ ); (b) Intensity ratio between both spectra as a function of the neutron wavelength.

\subsection{Final Remark: Beam Transport Efficiency}

A figure of merit that characterizes a good neutron transport is the brilliance transfer $(B T)$, defined as the neutron flux integrated over an area, wavelength and solid angle $\left(\mathrm{n} \cdot \mathrm{s}^{-1} \cdot \mathrm{cm}^{-2} \cdot \AA^{-1} \cdot \mathrm{sr}^{-1}\right)$ normalized to the same quantity close to the source [24]. Liouville's theorem states that $B T \leq 1$ in every passive optics system.

Figure 12 show the brilliance transfer, $B T$, as a function of wavelength from moderator to sample, defined by the ratio between the brilliance at the sample position and the brilliance at the source, $B$, in the same divergence range and spatial area [19]. Thus:

$$
\Phi / \Delta \Omega=B \times B T
$$

where $\Phi$ is the flux at the sample position and $\Delta \Omega$ is the solid angle transported from source to sample.

An adequate approach consists of assuming that the guided transport is perfect, the source solid angle transported to the sample $\Delta \Omega$ can be approximated as the product of the vertical $\left( \pm 2.5^{\circ}\right)$ and horizontal $\left( \pm 2.5^{\circ}\right)$ full width and a half maximum of the divergence distributions at the sample position (i.e., $\Delta \Omega=7.62 \mathrm{msr}$ ), yielding a maximum $B T$ achievable of approximately $\sim 16 \%$.

However, using the brilliance transfer to evaluate the goodness of a neutron transport system must be considered wisely. The estimated beam extraction efficiency, as such, tends to yield low values of this figure of merit when dealing with a source of small dimensions $[25,26]$. In this case, MIRACLES does not have a strict requirement in terms of beam divergence, and hence maximizing the phase space volume to collect more "useful" neutrons at the NBEX (thus to increase the final flux at the expense of a brilliance reduction) as well as improving the flux at the sample through beam focusing (at the expense of increasing the beam divergence) is a convenient strategy. 


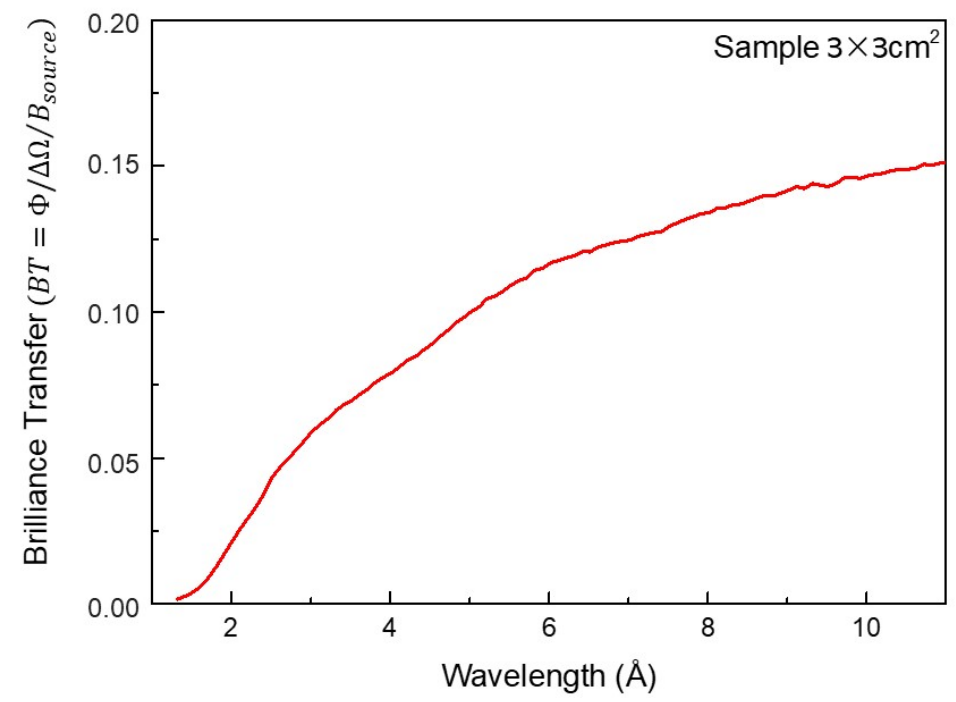

Figure 12. Brilliance transfer at the wavelength range of interest.

\section{Conclusions}

An updated conceptual design of the neutron delivery optics for MIRACLES, the time-of-flight backscattering instrument of the European Spallation Source, is presented. Calculation efforts aimed to efficiently transport cold neutrons of energy $E \lesssim 20 \mathrm{meV}$ (wavelength ranging from $\lambda=2$ to $\lambda=20 \AA$ ) from the ESS Butterfly moderator to the sample, through a distance of $162.5 \mathrm{~m}$. The new guide design also accounted for general engineering restrictions associated mainly with construction constraints and integration into the ESS facility. This factor primarily affects the beamline position and the dimensions of the beamline feedthroughs when crossing the shielding walls.

Whilst vertical layout takes advantage of the ballistic approach, the horizontal profile is determined by the high-energy resolution of the instrument and the selective transmission of cold neutrons. From here, calculations and design were optimized to maximize flux at the sample with a relaxed divergence distribution, rather than trying to reach high brilliance in small samples with low divergence. The results from these calculations can be summarized as follows:

- The vertical geometry of the neutron beam extraction section presents a strategy to improve the guide collection by modifying the elliptic profile of the neutron beam extraction, shifting the focus with respect to the cold center of the source, either horizontally or vertically. Both approaches yield an efficient larger aperture that conveys an improvement of the collection and transport of useful neutrons with respect to the use of standard elliptic profiles focused on such cold center. On the other hand, the horizontal geometry of the neutron beam extraction must comply with reduced dimensions at the pulse shaping chopper needed by the high-resolution performance of MIRACLES.

- The design of the curved section, needed to remove the background from high-energy neutrons, is based on a trade-off between the feasibility to include its whole length within the ESS common shielding area (bunker) and the maximization of the flux of cold neutrons at all the operational wavelength range. Such range includes not only the elastic wavelength/energy $(\lambda=6.27 \AA ; E=2.08 \mathrm{meV})$ but also higher energy neutrons (e.g., $\lambda \sim 3 \AA$ ), for which incident flux must be maximized for inelastic measurements (as well as quasielastic measurements using potentially $\mathrm{Si}(311)$ crystals in the future).

- $\quad$ Once the horizontal layout has dealt with the burden of high-resolution performance and high-energy neutron filtering, the guide geometry is modified to reduce the horizontal divergence distribution and its dependence on the neutron wavelength by means of a smooth expansion of the guide dimensions. This facilitates an efficient 
transport of the cold neutrons throughout the rest of the long beamline from the curve to the focusing guide sections $(\sim 100 \mathrm{~m})$.

- The focusing guide has been designed to efficiently focus the neutron beam for the whole operational wavelength band.

- Finally, a potential upgrade of the focusing guide has been proposed, consisting of an exchangeable final section allowed to reduce the beam footprint to close to $1 \mathrm{~cm}^{2}$, with a significant increase of the areal flux of up to $70 \%$.

With this new guide design that results in an overall optimization of the neutron flux throughout the selected cold wavelength range of interest, the MIRACLES beamline will be manufactured and integrated into the ESS facility. The work conducted here can be considered a benchmark for the more general optimization of beam transport systems for future ESS cold neutron spectrometers.

Author Contributions: Conceptualization, F.J.V. and D.M.R.; methodology, F.J.V., D.M.R., M.B. and H.N.B.; validation, F.J.V., D.M.R. and M.B.; formal analysis and investigation, all; writing-original draft preparation, F.J.V.; writing-review and editing, D.M.R., M.B. and H.N.B. All authors have read and agreed to the published version of the manuscript.

Funding: This research is partially funded by the Next Generation EU-Recovery and Resilience Facility (RRF).

Institutional Review Board Statement: Not applicable.

Informed Consent Statement: Not applicable.

Data Availability Statement: Not applicable.

Conflicts of Interest: The authors declare no conflict of interest.

\section{References}

1. Andersen, K.H.; Argyriou, D.; Jackson, A.; Houston, J.; Henry, P.; Deen, P.; Toft-Petersen, R.; Beran, P.; Strobl, M.; Arnold, T.; et al. The instrument suite of the European Spallation Source. Nucl. Instrum. Methods Phys. Res. Sect. A 2020, 957, 163402. [CrossRef]

2. Mamontov, E.; Herwig, K.W. A time-of-flight backscattering spectrometer at the Spallation Neutron Source, BASIS. Rev. Sci. Instrum. 2011, 82, 085109. [CrossRef]

3. Shibata, K.; Takahashi, N.; Kawakita, Y.; Matsuura, M.; Yamada, T.; Tominaga, T.; Kambara, W.; Kobayashi, M.; Inamura, Y.; Nakatani, T.; et al. The Performance of TOF near Backscattering Spectrometer DNA in MLF, J-PARC. JPS Conf. Proc. 2015, 8 , 036022 .

4. Lechner, R.E. Observation-time dependent structural information from quasielastic neutron scattering. Physica B 2001, 301, 83-93. [CrossRef]

5. Goracci, G.; Arbe, A.; Alegría, A.; García Sakai, V.; Rudić, S.; Schneider, G.J.; Lohstroh, W.; Juranyi, F.; Colmenero, J. Influence of Solvent on Poly(2-(Dimethylamino)Ethyl Methacrylate Dynamics in Polymer-Concentrated Mixtures: A Combined Neutron Scattering, Dielectric Spectroscopy, and Calorimetric Study. Macromolecules 2015, 48, 6724-6735. [CrossRef]

6. Miyatsu, S.; Kofu, M.; Nagoe, A.; Yamada, T.; Sadakiyo, M.; Yamada, T.; Kitagawa, H.; Tyagi, M.; García-Sakai, V.; Yamamuro, O. Proton dynamics of two-dimensional oxalate-bridged coordination polymers. Phys. Chem. Chem. Phys. 2014, 16, 17295. [CrossRef] [PubMed]

7. De Castro Lima, C.R.R.; Lima, R.J.S.; Machado, L.D.B.; Velasco, M.V.R.; Lakic, L.; Nordentoft, M.S.; Machuca-Beier, L.; Rudic, S.; Telling, M.T.F.; Garcia-Sakai, V.; et al. Human hair: Subtle change in the thioester groups dynamics observed by combining neutron scattering, X-ray diffraction and thermal analysis. Eur. Phys. J. Spec. Top. 2020, 229, 2825-2832. [CrossRef]

8. Tsapatsaris, N.; Lechner, R.E.; Marko, M.; Bordallo, H.N. Conceptual design of the time-of-flight backscattering spectrometer, MIRACLES, at the European Spallation Source. Rev. Sci. Instrum. 2016, 87, 085118. [CrossRef]

9. Tsapatsaris, N.; Willendrup, P.K.; Lechner, R.E.; Bordallo, H.N. From BASIS to MIRACLES: Benchmarking and perspectives for high-resolution neutron spectroscopy at the ESS. EPJ Web Conf. 2015, 83, 03015. [CrossRef]

10. Luna, P.; Bordallo, H.N.; Tsapatsaris, N.; Andersen, K.H.; Herranz, I.; Sordo, F.; Villacorta, F.J. Tailoring the energy resolution of MIRACLES, the time-of-flight-Backscattering spectrometer at the ESS: An updated proposal for the chopper cascade. Physica B 2019, 564, 64-68. [CrossRef]

11. Arai, M.; Zanini, L.; Andersen, K.H.; Klinkby, E.; Villacorta, F.J.; Shibata, K.; Nakajima, K.; Harada, M. The performance of ESS spectrometers in comparison with instruments at a short-pulse source. J. Neutron Res. 2020, 22, 71-85. [CrossRef]

12. Villacorta, F.J.; Bordallo, H.N.; Arai, M. A Pulse-Multiplication Proposal for MIRACLES, the Neutron TOF-Backscattering Instrument at the European Spallation Source. Quantum Beam Sci. 2021, 5, 2. [CrossRef] 
13. Lefmann, K.; Klenø, K.H.; Okkels Birk, J.; Hansen, B.R.; Holm, S.L.; Knudsen, E.; Lieutenant, K.; von Moos, L.; Sales, M.; Willendrup, P.K.; et al. Simulation of a suite of generic long-pulse neutron instruments to optimize the time structure of the European Spallation Source. Rev. Sci. Instrum. 2013, 84, 055106. [CrossRef]

14. Saroun, J.; Fenske, J.; Rouijaa, M.; Beran, P.; Navrátil, J.; Lukáš, P.; Schreyer, A.; Strobl, M. Neutron optics concept for the materials engineering diffractometer at the ESS. J. Phys. Conf. Ser. 2016, 746, 012011. [CrossRef]

15. Hilger, A.; Kardjilov, N.; Manke, I.; Zendler, C.; Lieutenant, K.; Habicht, K.; Banhart, J.; Strobl, M. Neutron guide optimisation for a time-of-flight neutron imaging instrument at the European Spallation Source. Opt. Express 2015, 23, 301-311. [CrossRef] [PubMed]

16. Andersen, K.H.; Bertelsen, M.; Zanini, L.; Klinkby, E.B.; Schonfeldt, T.; Bentley, P.M.; Saroun, J. Optimization of moderators and beam extraction at the ESS. J. Appl. Cryst. 2018, 51, 264-281. [CrossRef]

17. Willendrup, P.; Farhi, E.; Lefmann, K. McStas 1.7-A New Version of the Flexible Monte Carlo Neutron Scattering Package. Physica B 2004, 350, 735. [CrossRef]

18. Zanini, L.; Andersen, K.H.; Batkov, K.; Klinkby, E.B.; Mezei, F.; Schönfeldt, T.; Takibayev, A. Design of the cold and thermal neutron moderators for the European Spallation Source. Nucl. Instrum. Meth. Phys. Res. A 2019, 925, 33-52. [CrossRef]

19. Zendler, C.; Martin Rodriguez, D.; Bentley, P.M. Generic guide concepts for the European Spallation Source. Nucl. Instrum. Meth Phys. Res. A 2015, 803, 89-99. [CrossRef]

20. Zendler, C.; Nekrassov, D.; Lieutenant, K. An improved elliptic guide concept for a homogeneous neutron beam without direct line of sight. Nucl. Instrum. Meth. Phys. Res. A 2014, 746, 39-46. [CrossRef]

21. Maier-Leibnitz, H.; Springer, T. The use of neutron optical devices on beam-hole experiments on beam-hole experiments. J. Nucl. Energy A/B 1963, 17, 217-225. [CrossRef]

22. Martin Rodriguez, D.; DiJulio, D.D.; Bentley, P.M. Systematic study on the performance of elliptic focusing neutron guides. Nucl. Instrum. Meth. Phys. Res. A 2016, 808, 101-108. [CrossRef]

23. Ott, F. Focusing Optics for Neutrons. In Modern Developments in X-ray and Neutron Optics; Erko, A., Idir, M., Krist, T., Michette, A.G., Eds.; Springer: Berlin/Heidelberg, Germany, 2008; pp. 113-135.

24. Bertelsen, M.; Lefmann, K. Constraining neutron guide optimzations with phase-space considerations. Nucl. Instrum. Meth. Phys. Res. A 2016, 830, 313-324. [CrossRef]

25. Kajimoto, R.; Nakajima, K.; Nakamura, M.; Soyama, K.; Yokoo, T.; Oikawa, K.; Arai, M. Study of the neutron guide design of the 4SEASONS spectrometer at J-PARC. Nucl. Instrum. Meth. Phys. Res. A 2009, 600, 185-188. [CrossRef]

26. Nakajima, K.; Ohira-Kawamura, S.; Kikuchi, T.; Kajimoto, R.; Takahashi, N.; Nakamura, M.; Soyama, K.; Osakabe, T. Beamtransport optimization for cold-neutron spectrometer. EPJ Web Conf. 2015, 83, 03011. [CrossRef] 